\title{
Polymorphisms and ambiguous sites present in DNA sequences of Leishmania clones: Looking closer
}

\author{
Mariana Côrtes Boité*, Taíse Salgado de Oliveira, Gabriel Eduardo Melim Ferreira, Marcos Trannin, \\ Barbara Neves dos Santos, Renato Porrozzi, Elisa Cupolillo
}

Laboratório de Pesquisa em Leishmaniose, Instituto Oswaldo Cruz (IOC), Fundação Oswaldo Cruz (FIOCRUZ), Rio de Janeiro, Brazil

\section{A R T I C L E I N F O}

\section{Article history:}

Received 26 August 2013

Received in revised form 9 April 2014

Accepted 13 April 2014

Available online 21 April 2014

\section{Keywords:}

Leishmania

Polyclonal

Aneuploidy

Heterozygosis

\begin{abstract}
A B S T R A C T
In genetic studies of Leishmania parasites, co-dominant markers are chosen for their ability to detect heterozygous polymorphisms, to infer the occurrence of inbreeding and to resolve genetic variability. The majority of DNA sequence based reports perform conventional dye terminator cycle sequencing where perfectly ambiguous sites or double peaks in the chromatogram are interpreted as heterozygous strains. However, molecular peculiarities of the parasite such as aneuploidy, mixed populations and homologous recombination advise that data from regular DNA sequence analysis should be carefully evaluated. We report here a closer look at ambiguous sites observed in 6pgd DNA sequences obtained for a multilocus sequence analysis project on Leishmania (Viannia) strains. After comparing 286 DNA sequences from biological and molecular clones of six L. (Viannia) strains we could distinguish events that contribute to genetic variation in Leishmania (recombination, mutation, chromosomal mosaics). Also, the results suggest how diversity might not be completely revealed through regular DNA sequence analysis and demonstrate the importance for molecular epidemiology research to be aware of such possibilities while choosing samples for studies.
\end{abstract}

(c) 2014 Elsevier B.V. All rights reserved.

\section{Introduction}

The Leishmania parasite is the causative agent of the disease leishmaniasis, which affects millions of people annually and represents the third highest global burden of disease among the neglected tropical diseases. Despite observed morphological homogeneity across most specimens, more than 20 genetically distinct species have been described for the Leishmania genus. Phenotypic diversity can be observed between species, even among clones (Garin et al., 2001), which may be a causative agent in the presentation of different clinical forms of the disease and the variation in responses to conventional drug treatment. Therefore, ability to identify Leishmania species and to characterize genetic variants could increase physicians' ability to target treatment and therapy. With that in mind, many approaches have been developed, improved and applied aiming to contribute to population genetics, parasite identification and phylogeny. Among these approaches are multilocus enzyme electrophoresis (MLEE) (Cupolillo et al., 1994) and molecular markers based on DNA

\footnotetext{
* Corresponding author. Address: Av Brasil, 4365, Pavilhão Leonidas Daene sala 509, Manguinhos, Rio de Janeiro, RJ, Brazil. Tel.: +55 2138658226.

E-mail address: boitemc@ioc.fiocruz.br (M.C. Boité).
}

sequence analysis, such as multilocus sequence analysis (MLSA) (Zemanova et al., 2007; El Baidouri et al., 2013; Boité et al., 2012) and multi locus microsatellite typing (Alam et al., 2009; Oddone et al., 2009; Rougeron et al., 2010).

Traditionally, co-dominant markers used for these genetic based analyses are chosen by their ability to detect heterozygous polymorphisms, infer the presence of inbreeding, and determine genetic variability. The majority of DNA sequence based protocols utilize conventional dye terminator cycle sequencing, which assume standard procedures for interpreting inconsistencies in results, such as double vs single peaks. In MLSA sequences, double peaks in the chromatogram are normally considered to result from heterozygous strains. However, four major aspects of Leishmania biology raise concern over the interpretation of such DNA sequence based analysis. First, Leishmania presents mosaic aneuploidy in vitro (Sterkers et al., 2010; Rogers et al., 2011), which has also been detected in clinical isolates (Rogers et al., 2011). Mosaic aneuploidy creates cells with different karyotype contents and may result in overlapping the reads of different nucleotides, actually present in different cells, leading to misinterpretation or loss of information in the sequence analysis. Next, the occurrence of polyclonality and infrapopulation mixture in Leishmania isolates could cause the observation of more than one sequence in the 
chromatogram. This can occur simply by natural mixing or can be created by karyotype diversity (Dujardin et al., 2007) generated after asymmetric chromosomal allotment (ACA) (Sterkers et al., 2012). Third, clonal propagation is considered the predominant mode of reproduction of this parasite. This phenomenon may overlook cryptic sexuality and could explain the presence of the double peak rather than being representative of the clone. Finally, the clonal concept in Leishmania is more plausible from a biological point of view rather than a genetic one (Tibayrenc et al., 1990; Tibayrenc and Ayala, 2012).

Given that misinterpretation of sequencing results may have an important impact on all Leishmania typing and characterization studies, we investigated ambiguous sites represented by double peaks in 6pgd DNA sequences obtained by MLSA for Leishmania (Viannia) strains (Boité et al., 2012) to observe how the above described parasite characteristics may interfere in DNA sequences generated for genetic variability analysis.

\section{Material and methods}

\subsection{Strains, biological cloning and MLEE characterization}

Six L. (Viannia) strains (Table 1) presenting double peaks in previously obtained 6pgd DNA sequences (Boité et al., 2012) were retrieved from the Coleção de Leishmania do Instituto Oswaldo Cruz (CLIOC - WDCM 731). More details about the samples can be obtained by accessing the CLIOC catalogue (http://clioc.fiocruz.br). The parasites were cultivated in Schneider's medium and the culture volume divided for both procedures: biological cloning and direct DNA isolation. For biological cloning $10^{4}$ parasites in $600 \mu \mathrm{l}$ of Schneider's medium were added to $2.4 \mathrm{ml}$ of Low Melting Point Agarose (Sigma) 1\% warmed solution. The mixture was distributed over a Petri Plate containing NNN medium. After 7 days, the colonies were harvested and cultured separately in tubes containing biphasic medium (NNN + Schneider's) and processed for DNA isolation and MLEE assay. Enzymatic MLEE systems were assayed for the characterization of biological clones as previously described (Cupolillo et al., 1994).

\subsection{Molecular procedures and analysis}

DNA purification, PCRs for the 6pgd locus and PCR product purification was carried out as described by Boité et al. (2012). Sequences were obtained from two parasite states: non cloned culture (NC) and culture from biological clone (BC) as pointed in Fig. 1; and through two distinct approaches: PCR products subjected to direct sequencing (DS) and to molecular cloning followed by sequencing (MC) (Fig. 1A). These conditions combined generated the following DNA sequences: non-cloned directly sequenced (NCDS), biological clones directly sequenced (BCDS) and biological clones subjected to molecular cloning (BC_MC) (Fig. 1B).

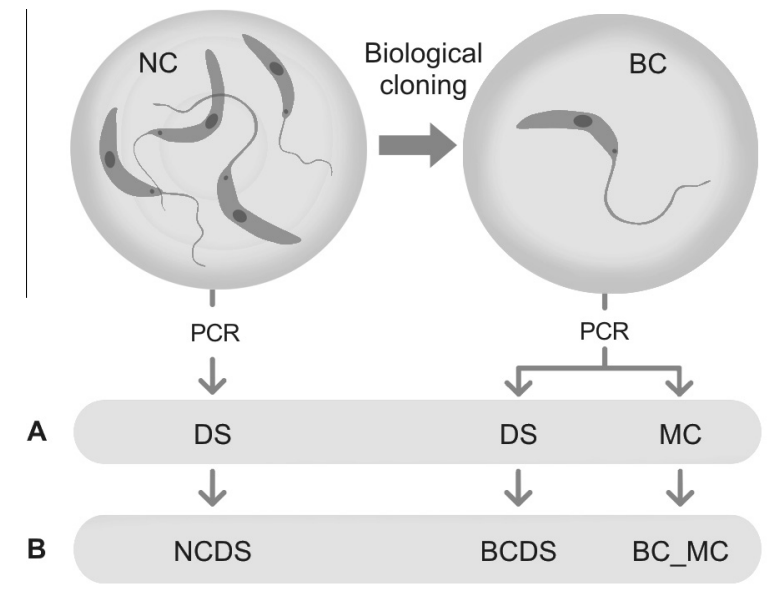

Fig. 1. Procedures to obtain the DNA sequences compared in the present study. Cultures used for DNA isolation: non-cloned (NC) and after biological cloning (BC). $A=P C R$ products obtained from each culture either subjected to direct sequencing (DS) or molecular cloning (MC). B = Sequences obtained from NC and BC after MC or DS: the "non-cloned directly sequenced" (NCDS) represents a sequence obtained from the PCR product of DNA isolated from a non-cloned population of cells; the "biological clone directly sequenced" (BCDS) represents a sequence obtained from the PCR product of DNA isolated from cloned cultures; the "biological clone_molecular clone" (BC_MC) represents a sequence obtained after molecular cloning of the PCR product of DNA isolated from cloned cultures.

Purified PCR products from BC were molecularly cloned using pGEM $^{\circledR}$-T Easy Vectors System (Promega) and sequences obtained in an ABI 3730 DNA Sequencer by the PDTIS (FIOCRUZ, Rio de Janeiro, Brazil) sequencing service (Otto et al., 2008). Consensus sequences were generated and edited in Phred/Phrap/Consed Version: 0.020425.c from forward and reverse strands. Only sequence segments with Phred values above twenty were used for contig construction. The chromatogram was manually tracked for double peaks (equivalent presence of nucleotides, suggesting a 1:1 proportion of alleles) in at least one strand, and the respective sites coded using the standard IUPAC for combinations of two or more bases.

Contigs from all samples were manually assembled and aligned in MEGA5 software (Tamura et al., 2011). All new sequences obtained were compared with those from Boité et al. (2012) named here as "previous NCDS".

Recombination analysis was performed in RDP (Martin et al., 2010), using different recombination detection algorithms, with other L. (Viannia) sequences from Boité et al. (2012) and sequences available in GenBank ${ }^{\circledR}$.

\section{Results and discussion}

Results demonstrated that Leishmania parasite biology may play a significant role in the outcome of sequence analysis. We found

Table 1

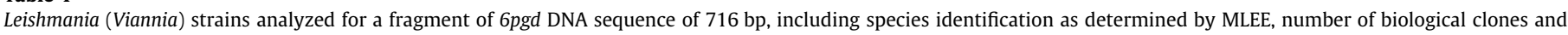
molecular clones, different alleles (sequence types) obtained and type of event observed.

\begin{tabular}{|c|c|c|c|c|c|c|}
\hline Species by MLEE & $\mathrm{IOC} / \mathrm{L}$ & Total of DNA sequences compared" & BCDS & MC & Sequence types ${ }^{* *}$ & Events observed \\
\hline L. braziliensis & 918 & 50 & 8 & 40 & 10 & Hot spot sites \\
\hline L. braziliensis & 2538 & 52 & 9 & 41 & 9 & Homologous recombination \\
\hline L. braziliensis & 2823 & 134 & 20 & 112 & 14 & Infrapopulation of nonequivalent heterozygous cells \\
\hline L guyanensis & 2966 & 7 & 5 & 0 & 2 & ACAs; polymorphisms \\
\hline L. lainsoni/L. naiffi & 2490 & 23 & 4 & 17 & 3 & Monoclonal heterozygous \\
\hline L. guyanensis & 2957 & 20 & 4 & 15 & 2 & ACAs \\
\hline
\end{tabular}

MLEE = multilocus enzyme electrophoresis; $\mathrm{BCDS}=$ biological clones directly sequenced; $\mathrm{MC}=$ molecular clones; ACAs = asymmetric chromosomal allotments.

* Including the previous and recent NCDS.

** Sequence types were determined considering a 6pgd gene region as described previously (Boité et al., 2012). 
that mosaic aneuploidy generated by ACA occurring during parasite culture played an important part in the interpretations of results. These results have a considerable impact on past and future molecular epidemiologic studies of leishmaniasis since we can no longer assume heterozygosis as the unique cause of ambiguous sites in sequencing based methods.

\subsection{Loss of ambiguous site by asymmetric chromosomal allotments (ACA)}

The double peak previously detected for two strains IOC/L 2957 and 2966 in site 602 was no longer observed, and NCDS and BCDS were identical, suggesting a monoclonal population (Table 2). The loss of heterozygous cells and the alternative allele once detected can be explained by ACAs during the axenic culture (Sterkers et al., 2012). The clonal propagation of the parasites presenting the homozygous chromosomal arrangement might have leaden to the detection of only DNA sequences without the double peaks in the chromatogram. It is not possible, however, to be certain about ploidy of the cell since ACAs explain the monomorphic DNA sequences among BCDS and MC, but ACAs also generates karyotype diversity.

In addition to the double peak site, polymorphisms were noticed between the previous sequence and the new sequences (sites 640,672, 685, Table 2). These polymorphisms could be the result of point mutations during the culture procedure. Double peak and polymorphic sites, associated with preponderant clonal evolution (PCE) (Tibayrenc and Ayala, 2013) represents one more complicating factor in Leishmania population structure analysis; nevertheless some strictly sexual organisms might also challenge models dependent on whether ability to prevent whole chromosome elimination is present (Benatti et al., 2010).

3.2. Less frequent alleles can only be detected after molecular cloning in conventional dye terminator sequencing methods

The previously observed double peak for the strain IOC/L 2823 represented an equivalent proportion of nucleotides (1:1) but

Table 2

Sequence polymorphisms observed in a 6pgd gene region for non-cloned culture, and for biological and molecular clones of six Leishmania strains.

\begin{tabular}{|c|c|c|c|c|c|c|c|c|c|c|c|c|c|c|c|c|c|c|c|c|c|c|c|c|c|c|c|c|c|c|}
\hline \multirow[t]{4}{*}{ IOC } & \multirow{4}{*}{$\begin{array}{l}\text { Sequence origin } \\
\text { (origin) number of sequences }\end{array}$} & \multicolumn{29}{|c|}{ Site position } \\
\hline & & & & & & 1 & 1 & 1 & 1 & 2 & 2 & 2 & 3 & 3 & 3 & 3 & 4 & 4 & 4 & 4 & 5 & 5 & 5 & 5 & 6 & 6 & 6 & 6 & 6 & 6 \\
\hline & & 4 & 4 & 6 & 8 & 0 & 0 & 0 & 8 & 0 & 1 & 3 & 1 & 2 & 5 & 9 & 0 & 4 & 6 & 6 & 3 & 5 & 6 & 7 & 0 & 3 & 4 & 6 & 7 & 8 \\
\hline & & 2 & 5 & 4 & 5 & 1 & 2 & 6 & 9 & 7 & 9 & 2 & 9 & 9 & 8 & 1 & 0 & 0 & 0 & 6 & 8 & 2 & 4 & 8 & 2 & 0 & 0 & 6 & 2 & 5 \\
\hline \multirow[t]{2}{*}{ L 2966} & Previous NCDS & & & & & & & & & & & & & & & & & & & & & & & & $\mathbf{R}$ & & A & & G & G \\
\hline & $(\mathrm{NCDS})_{1} ;(\mathrm{BCDS})_{5}$ & & & & & & & & & & & & & & & & & & & & & & & & A & & G & & C & $\mathrm{T}$ \\
\hline \multirow[t]{2}{*}{ L 2957} & Previous NCDS & & & & & & & & & & & & & & & & & & & & & & & & $\mathbf{R}$ & & & & & \\
\hline & $(\mathrm{NCDS})_{1} ;(\mathrm{BCDS})_{4} ;(\mathrm{MC})_{17}$ & & & & & & & & & & & & & & & & & & & & & & & & A & & & & & \\
\hline \multirow[t]{14}{*}{ L 2823} & Previous NCDS & & $\mathrm{T}$ & & $\mathrm{C}$ & A & & $\mathrm{C}$ & $\mathrm{T}$ & $\mathrm{C}$ & A & A & $\mathrm{T}$ & $\mathrm{T}$ & $\mathrm{T}$ & & $\mathrm{T}$ & & $\mathrm{Y}$ & & A & A & A & A & & A & & A & & \\
\hline & $(\mathrm{NCDS})_{1} ;(\mathrm{BCDS})_{17} ;\left(\mathrm{BC} \_\mathrm{MC}\right)_{90}$ & & . & & . & . & & . & . & . & . & . & . & . & . & & . & & $\mathrm{T}$ & & . & . & . & . & & . & & . & & \\
\hline & $(\mathrm{BCDS})_{3} ;\left(\mathrm{BC} \_\mathrm{MC}\right)_{1}$ & & $\mathrm{C}$ & & . & . & & . & . & . & . & . & . & . & . & & . & & $\mathrm{T}$ & & . & . & . & . & & . & & . & & \\
\hline & $\left(\mathrm{BC} \_\mathrm{MC}\right)_{2}$ & & . & & . & . & & . & . & . & G & . & . & . & . & & . & & $\mathrm{T}$ & & . & . & . & . & & . & & . & & \\
\hline & $\left(\mathrm{BC} \_\mathrm{MC}\right)_{2}$ & & . & & . & . & & $\mathrm{T}$ & . & . & . & . & . & . & . & & . & & $\mathrm{T}$ & & . & G & . & . & & . & & . & & \\
\hline & $\left(\mathrm{BC} \_\mathrm{MC}\right)_{2}$ & & . & & . & . & & . & . & . & . & . & . & . & . & & . & & $\mathrm{T}$ & & . & . & . & G & & . & & . & & \\
\hline & $\left(\mathrm{BC} \_\mathrm{MC}\right)_{3}$ & & . & & $\mathrm{T}$ & . & & . & . & . & . & . & . & . & $\mathrm{C}$ & & A & & $\mathrm{T}$ & & . & . & . & . & & G & & . & & \\
\hline & $\left(\mathrm{BC} \_\mathrm{MC}\right)_{3}$ & & . & & . & . & & . & . & . & . & G & . & . & . & & . & & $\mathrm{T}$ & & . & . & . & . & & . & & . & & \\
\hline & $\left(\mathrm{BC} \_\mathrm{MC}\right)_{3}$ & & . & & . & . & & . & . & . & . & . & $\mathrm{C}$ & C & . & & . & & $\mathrm{T}$ & & . & . & . & . & & . & & . & & \\
\hline & $\left(\mathrm{BC} \_\mathrm{MC}\right)_{1}$ & & . & & . & . & & . & . & . & . & . & . & . & . & & . & & C & & . & . & . & . & & . & & . & & \\
\hline & $\left(\mathrm{BC} \_\mathrm{MC}\right)_{2}$ & & . & & . & . & & . & C & $\mathrm{T}$ & . & . & . & . & . & & . & & $\mathrm{T}$ & & . & . & . & . & & . & & . & & \\
\hline & $\left(\mathrm{BC} \_\mathrm{MC}\right)_{2}$ & & . & & . & $\mathrm{T}$ & & . & . & . & . & . & . & . & . & & . & & $\mathrm{T}$ & & . & . & . & . & & . & & G & & \\
\hline & $\left(\mathrm{BC} \_\mathrm{MC}\right)_{1}$ & & . & & . & . & & . & . & . & . & . & . & . & . & & . & & $\mathrm{T}$ & & G & . & G & . & & . & & . & & \\
\hline & $\left(\mathrm{BC} \_\mathrm{MC}\right)_{1}$ & & . & & . & . & & . & . & . & . & . & . & . & . & & . & & $\mathrm{T}$ & & G & . & . & . & & . & & . & & \\
\hline \multirow[t]{9}{*}{ L 2538} & Previous NCDS; $(\mathrm{BCDS})_{3}$ & & & $\mathrm{R}$ & & & & & & & & & & & & $\mathrm{S}$ & & & $\mathrm{Y}$ & $\mathrm{Y}$ & & & & & & & & & & \\
\hline & $(\mathrm{NCDS})_{1} ;(\mathrm{BCDS})_{4}$ & & & A & & & & & & & & & & & & . & & & . & . & & & & & & & & & & \\
\hline & $(\mathrm{BCDS})_{2}$ & & & . & & & & & & & & & & & & . & & & . & $\mathrm{C}$ & & & & & & & & & & \\
\hline & $\left(\mathrm{BC} \_\mathrm{MC}\right)_{14}$ & & & G & & & & & & & & & & & & $\mathrm{C}$ & & & $\mathrm{C}$ & $\mathrm{C}$ & & & & & & & & & & \\
\hline & $\left(\mathrm{BC} \_\mathrm{MC}\right)_{5}$ & & & G & & & & & & & & & & & & G & & & $\mathrm{T}$ & $\mathrm{T}$ & & & & & & & & & & \\
\hline & $\left(\mathrm{BC} \_\mathrm{MC}\right)_{13}$ & & & A & & & & & & & & & & & & G & & & $\mathrm{T}$ & $\mathrm{T}$ & & & & & & & & & & \\
\hline & $\left(\mathrm{BC} \_\mathrm{MC}\right)_{7}$ & & & A & & & & & & & & & & & & $\mathrm{C}$ & & & $\mathrm{T}$ & $\mathrm{C}$ & & & & & & & & & & \\
\hline & $\left(\mathrm{BC} \_\mathrm{MC}\right)_{2}$ & & & A & & & & & & & & & & & & $\mathrm{C}$ & & & $\mathrm{C}$ & $\mathrm{C}$ & & & & & & & & & & \\
\hline & $\left(\mathrm{BC} \_\mathrm{MC}\right)_{1}$ & & & A & & & & & & & & & & & & $\mathrm{C}$ & & & $\mathrm{T}$ & $\mathrm{T}$ & & & & & & & & & & \\
\hline \multirow[t]{4}{*}{ L 2490} & Previous NCDS; (NCDS $)_{1} ;(\mathrm{BCDS})_{4}$ & & & & & & $\mathrm{R}$ & & & & & & & & & & & $\mathrm{K}$ & & & & & & & & & & & & \\
\hline & $\left(\mathrm{BC} \_\mathrm{MC}\right)_{9}$ & & & & & & A & & & & & & & & & & & G & & & & & & & & & & & & \\
\hline & $\left(\mathrm{BC} \_\mathrm{MC}\right)_{1}$ & & & & & & G & & & & & & & & & & & G & & & & & & & & & & & & \\
\hline & $\left(\mathrm{BC} \_\mathrm{MC}\right)_{7}$ & & & & & & G & & & & & & & & & & & $\mathrm{T}$ & & & & & & & & & & & & \\
\hline \multirow[t]{10}{*}{ L 918} & Previous NCDS; (NCDS) 1 & $\mathrm{~T}$ & & G & & & & & & & & & & & & $\mathrm{S}$ & & & $\mathrm{Y}$ & $\mathrm{Y}$ & & & & & & & & & & \\
\hline & $(\mathrm{BCDS})_{3}$ & . & & A & & & & & & & & & & & & . & & & . & . & & & & & & & & & & \\
\hline & $(\mathrm{BCDS})_{1}$ & C & & A & & & & & & & & & & & & . & & & $\mathrm{C}$ & . & & & & & & & & & & \\
\hline & $(\mathrm{BCDS})_{1}$ & C & & . & & & & & & & & & & & & . & & & $\mathrm{C}$ & . & & & & & & & & & & \\
\hline & $(\mathrm{BCDS})_{1}$ & . & & C & & & & & & & & & & & & . & & & . & . & & & & & & & & & & \\
\hline & $(\mathrm{BCDS})_{2}$ & . & & . & & & & & & & & & & & & . & & & $\mathrm{C}$ & $\cdot$ & & & & & & & & & & \\
\hline & $\left(\mathrm{BC} \_\mathrm{MC}\right)_{17}$ & . & & A & & & & & & & & & & & & G & & & $\mathrm{T}$ & $\mathrm{T}$ & & & & & & & & & & \\
\hline & $\left(\mathrm{BC} \_\mathrm{MC}\right)_{5}$ & . & & A & & & & & & & & & & & & $\mathrm{C}$ & & & $\mathrm{C}$ & $\mathrm{C}$ & & & & & & & & & & \\
\hline & $\left(\mathrm{BC} \_\mathrm{MC}\right)_{17}$ & . & & . & & & & & & & & & & & & $\mathrm{C}$ & & & $\mathrm{C}$ & C & & & & & & & & & & \\
\hline & $\left(\mathrm{BC} \_\mathrm{MC}\right)_{1}$ & . & & . & & & & & & & & & & & & G & & & $\mathrm{T}$ & $\mathrm{T}$ & & & & & & & & & & \\
\hline
\end{tabular}

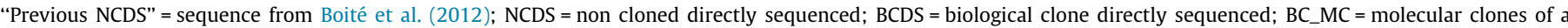
biological clone; $\mathrm{R}=\mathrm{A}$ (adenine) or $\mathrm{G}$ (guanine); $\mathrm{Y}=\mathrm{C}$ (citosine) or $\mathrm{T}$ (timine); $\mathrm{S}=\mathrm{G}$ or $\mathrm{C} ; \mathrm{K}=\mathrm{G} / \mathrm{T}$. 
was no longer identified among BCDS (Table 2, site 460); nonetheless one MC presented the alternative nucleotide "C". From that is possible to infer a change has occurred in allele's proportion, and only biological and molecular cloning allowed both nucleotide possibilities to be detected.

The heterozygous concept applied for Leishmania is the 1:1 nucleotides ratio in chromatogram. However, for polyploidy organisms, it is appropriated to determine a heterozygous individual by the identification of different copies for a given target gene or locus (van Belkum et al., 2001). The present result represents exactly such situation. The detection of less frequent alleles in non-cloned culture can be performed either through molecular cloning or, very cautiously, in chromatogram (Fig. 2). For the last option, however, the approach should be first validated since the smaller peak can be misinterpreted as background. Because of this, in the present study we only considered the sequences from molecular cloning to determine different copies of the targeted allele.

Aside the ambiguous sites targeted as the main objective of study, polymorphisms were also noticed along the alignment of all sequences obtained for this strain. In site 45 , for instance, three BCDS and one BC_MC differ from their correspondent MCs and BC (Table 2). This suggests that biological clones represent a heterozygous infrapopulation, and the alternative allele (T) is among the respective BC_MC, but not in equal proportion to generate overlapped peaks in BCDS or NCDS.

The polymorphisms can be explained either by: (i) point mutations, which have generated infrapopulation during the culture or (ii) initial aneuploidy, with different heterozygote cells: the sequences with the polymorphism in position 45 would represent the least frequent alleles and therefore could be detected only after biological and molecular cloning. Although mutations might occur frequently, option (ii) is more plausible because it is corroborated by the detection of MC presenting alleles different from their corresponding BCDS. Homozygous cells might also be present (after MC many presented just one allele). Nonetheless these findings demonstrate how less frequent alleles might often be undetected or excluded from DNA sequence based analysis. It also points that the removal of samples presenting multiple peaks would not avoid this bias (Camara et al., 2010).

\subsection{Detection of polyclonal heterozygous sample and homologous recombination}

Previous NCDS of IOC/L 2538 presented four double peaks and identical sequence types were observed, after biological cloning, among three BCDS (Table 2). However, the pattern was not kept for all other sequences: the NCDS differed in site 64 from the previous obtained sequence; the BCDS were either (i) identical to the previous sequence; (ii) identical to the NCDS or (iii) a third variation, with no double peak in the site 466 (Table 2). These results suggest at least three possible heterozygous cell populations occur within the IOC/L 2538 strain. However, when each site is observed, only positions 391 and 460 appear as classical heterozygous, with the possible nucleotides detected among the MCs. Such pattern would be expected for all sites since they are located in the same locus.

For sequence types from case (ii), the corresponding MCs presented both nucleotide possibilities in the four sites, including position 64, which had no double peak. Therefore, it might represent a heterozygous infrapopulation in the sample, containing alleles with different nucleotides only in position 64 . This confirms

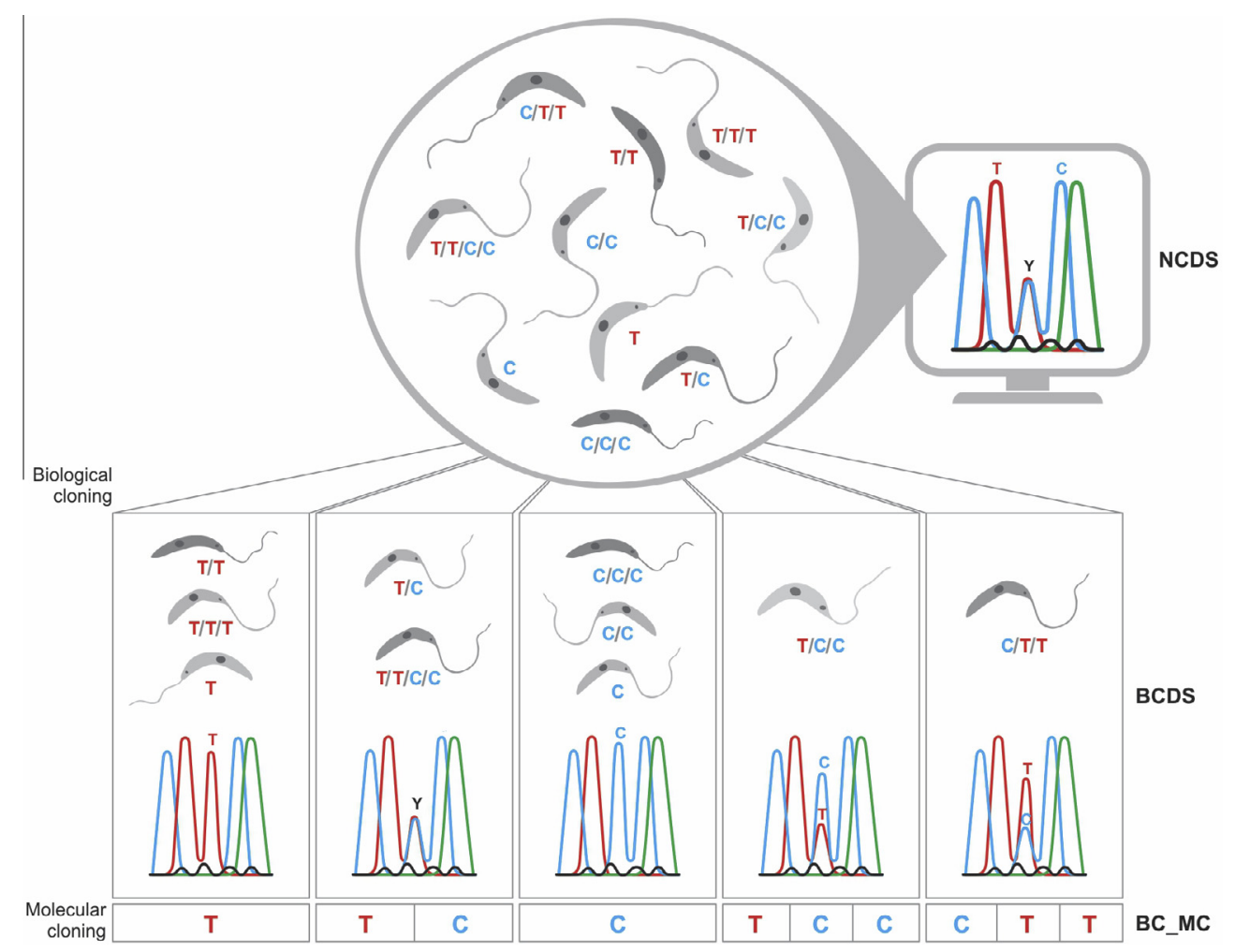

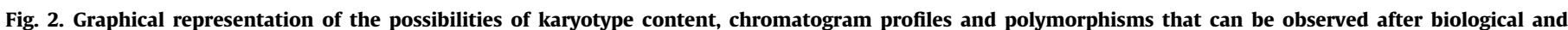

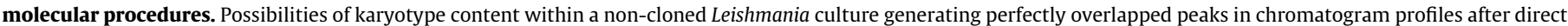

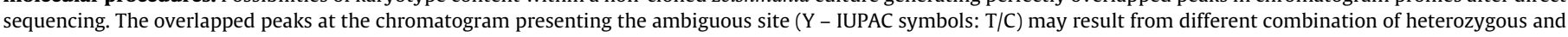

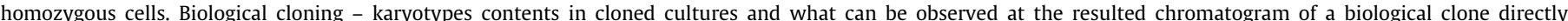

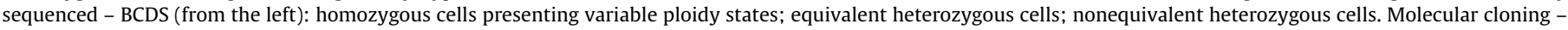
polymorphisms detected at the ambiguous sites after sequencing DNA isolated from biological clones and submitted to molecular cloning (BC_MC). 
a third heterozygous allele within the culture observed only after $\mathrm{BC}$ and MC.

Upon molecular cloning in a diploid organism, the four ambiguous sites could generate at most 16 possible haplotypes. Here, a total of six different haplotypes were detected among $42 \mathrm{MCs}$, obtained from nine BCs from the same strain IOC/L 2538. This number of alleles demonstrates the intra-strain sequence type diversity. Although PCR recombination and point mutation cannot be excluded, the origin of the supernumerary heterozygous alleles could also be consequence of homologous recombination. In most organisms, the primary function of homologous recombination is to allow genome protection by the faithful repair of DNA doublestrand breaks and search for sequence homology. However, Trypanosoma brucei and Leishmania use homologous recombination as a beneficial mechanism for antigenic variation (Boothroyd et al., 2009) or for drug resistance (Ubeda et al., 2008), respectively.

These results reflect the aneuploidy state and homologous recombination. As an aneuploidy organism, a gene previously described as one-copy can in fact present a variable number of copies. Moreover, even between BCs, ACAs do occur during parasite growth (mitosis). Thus, if a BC is a heterozygous cell initially, this state may not be sustained during the necessary period of culture to obtain DNA. Nevertheless, as pointed out by Sterkers et al. (2012), the alleles will still be present in the same initial proportion (the process is stable instead of fluctuating) although the number of heterozygous cells may decrease. This means if the heterozygote was not detected at first, it will be after MC (see point 3.2).

\subsection{Monoclonal heterozygous strain profiling in DNA sequences cannot exclude karyotype diversity}

The strain IOC/L 2490 preserved the two double peaks in NCDS and BCDS (Table 2). The NCDS and all four BCDS identical to the previous sequence suggest, therefore, a monoclonal heterozygous strain. However, the clonal concept in Leishmania is only suitable from a biological point of view, but not genetically (Dujardin et al., 2007) since karyotype diversity is generated from one parasite cell (Sterkers et al., 2012, 2011). Indeed, the clonal theory for Leishmania does not refer to any precise cytological mechanism, but instead to the genetic consequences of clonality. In the present study we cannot guarantee the cells present the same karyotype, but it is possible to guarantee the equivalent proportion of alleles through the perfect 1:1 overlapping peaks observed (Fig. 2). Considering the two polymorphic sites (Table 2) and recombination occurrence, four possible haplotypes at most could be detected for a diploid cell. Three alleles were detected within $17 \mathrm{MCs}$, suggesting more than two homologous chromosomes are present.

\subsection{The double peaks and polymorphisms presented coincident sites between the strains analyzed}

Strain IOC/L 918 NCDS and previously NCDS were identical, with the three double peaks still present (Table 2). For the BCDS, two sites $(391,466)$ kept the ambiguity. Two other sites $(42,64)$, not double peaks initially, were included in the analysis because, curiously, among the respective MCs both nucleotide alternatives were observed. Moreover, these sites were also double peaks for other strains (IOC/L 2494 and 2538).

Five haplotypes (Table 2) where detected among the eight BCDS. This may represents the occurrence of populations with different heterozygous karyotypes. Additionally, it suggests the polymorphic sites, which were double peaks for other strains but not for IOC/L 918, might be related to hot spots of mutation/ recombination.

\subsection{Recombination detection}

After RDP analysis including 175 L. (Viannia) non-cloned sequences plus all 286 sequences obtained in the present study, one recombination event was detected for one Leishmania braziliensis strain (IOC/L 3089, from Bahia, Northeastern Brazil). The major and minor parental populations indicated were, respectively, IOC/L 3072 (L. braziliensis, from Rio de Janeiro), and the BCDS and NCDS (which were identical) of IOC/L 2490 Leishmania naiffi/ Leishmania lainsoni - (hybrid profile in 6PGDH isoenzyme) by MaxChi and 3Seq algorithms $(P<0.05)$. The breakpoint beginning/end was wide enough to encompass almost the whole sequence, but the MaxChi graph representation indicates a window between positions 350 and 492, which includes the main polymorphic and double peaks sites evaluated in the present study.

Recombination is usually hard to detect within species, mainly because of low inter-strain diversity, or apparent low diversity due to inappropriate sampling (Prugnolle and De Meeus, 2010). However, some authors were able to present such data: population genetics studies have described inbreeding in L. braziliensis as well as in other Leishmania species (Rougeron et al., 2010; Ferreira et al., 2012). Recombination signals appear also in MLSA approaches (Boité et al., 2012) and in natural hybrids that were widely described (Akopyants et al., 2009; Belli et al., 1994; Brito et al., 2009) or even created in vitro (Coelho et al., 2012). RDP software recognizes IUPAC symbols, enhancing the ability of the algorithms to detect recombination. The possibility of PCR recombination cannot be excluded, but random PCR repetition of some sequences (data not shown) validated the polymorphisms detected. The present results indicate that recombination may occur between specific clones, and the molecular and biological cloning can contribute to the detection of a specific recombinant allele. Well-structured clonal complexes in Leishmania (Boité et al., 2012), could be the consequence of homologous recombination, as described in bacteria (Gonzalez-Gonzalez et al., 2013; Paul et al., 2013; Jinkerson et al., 2013) and other microorganisms (Vink et al., 2011).

\section{Further comments}

The main concern that has driven this study is the discussion regarding intra strain variation detected here in DNA sequence based analysis in Leishmania and elsewhere in Trypanosoma cruzi (Camara et al., 2010). The exact determination of allele frequency in individuals and, eventually, in populations is essential in association studies (Tibayrenc and Ayala, 2012, 2013); therefore, the quantitative accuracy of genotyping is critical. Multiple peaks in chromatograms, for instance, might point to: (i) different clones in the sample; (ii) heterozygotes or both. After the present results we observe that to exclude strains which present multiple peaks from the analysis is not the best way to deal with these facts since such diversity might be present even when the multiple peaks are not detected.

The occurrence of polyclonal samples is a constant if one considers the aneuploidy state described for Leishmania. The ACAs during mitosis seem to be quite frequent, generating daughter cells with different karyotype profiles, even after cellular cloning (infrapopulation) (Sterkers et al., 2012). Therefore, the (genetic) clonal concept is quite intricate to be applied in this parasite (Tibayrenc and Ayala, 2012). We demonstrate here that there is DNA sequence diversity within one strain kept in culture and/or cryopreserved; and changes can be detected along time, in different DNA batches. The allele frequency, however, usually does not change within the mosaic population. If multiple peaks are detected once and the two most prominent considered, that means the most frequent alleles (prevalent infrapopulation in the sample) are being distinguished. 
Nevertheless, as presented in this project, some alleles will only be observed after BC and MC.

In terms of allele frequency, DNA sequence based approaches are able to reflect (partially) the biological reality, especially if there is a good sample representation in terms of diversity and geographic origin. A strain will still appear as a 'heterozygote' when global analysis methods are used because all the alleles present at first (in a real heterozygous cell) are still present in the population. This process seems to be stable, so, despite the mosaic of cells, a pattern can still be determined as representative for that population. From a population genetics point of view, if a heterozygous signal is detected, it does represent heterozygous strains at some point and reflects the two most frequent alleles in that sample. Nevertheless, the real diversity will always be underestimated (Prugnolle and De Meeus, 2010) because some alleles will remain undetected. The bias of underestimated diversity could be diminished through a proper representation of the circulating strains, such that the greatest number of different alleles would be detected. The differences found between NCDS and the previous sequences are the most disquieting findings in terms of DNA based genetic studies.

The ratio of overlapping peaks in electropherograms can vary in polyploidy genomes (Fig. 2) (Rickert et al., 2002). In tetraploid chromosomes, for instance, five allelic ratios are distinguishable: $4: 0,3: 1,1: 1,1: 3$, and $0: 4$. The present targeted $6 p g d$ sequences, after Genome Blast (http://www.ncbi.nlm.nih.gov/) and search in http://tritrypdb.org, were described as located in chromosome 35 (Rogers et al., 2011) and 34/20 (chromosome fusion), respectively. Either way, these chromosomes are considered non-supernumerary in L. braziliensis, which would mean a triploid state previously described (Rogers et al., 2011). Only perfectly double peaks were considered in the $L$. (Viannia) strains analyzed here suggesting a $1: 1$ proportion of alleles, which is an unexpected result for triploids $(1: 2 ; 3: 0)$. If the triploid state is indeed real for $L$. (Viannia) strains, the results could reflect either the presence of other gene copies or the occurrence of ACAs, e.g. triploid heterozygous cells with an equal proportion of the alleles in the population (Fig. 2). Thus the strain would still appear as a "perfect monoclonal heterozygote" when sequencing methods are used.

Strains with a non-equivalent proportion for different alleles (such as 3:1 - multiple peaks) are usually analyzed as homozygous favoring the most prevalent allele. On the other hand, those strains considered heterozygous probably were either real heterozygotes at some point, or are composed by homozygous cells presenting different alleles in exactly equivalent proportions for the locus under study. If the last option occurs, it is unlikely to be due to occurrence of mixed populations; the most reasonable explanation is occurrence of cells under ACA events, descendent from a real heterozygote. Even for clones, ACAs do occur during parasite replication (mitosis). So, if a clone is a heterozygous cell initially, such a situation may not be sustained during the period of culture required to obtain DNA.

\section{Conclusions}

Results obtained after DNA sequence based analysis reflect events that contribute to genetic variation in Leishmania (recombination, mutation, chromosomal mosaics). The DNA sequences variety observed within the same strain demonstrates how diversity might not be completely represented through regular DNA sequence analysis and signal the importance for molecular epidemiology research to be aware of such possibilities while choosing the samples for studies. Moreover, to exclude samples with multiple peaks from analysis is not the best way to deal with the occurrence of infra-populations in Leishmania because such a phenomenon occurs frequently. Instead, tests based on diploidy, often used for Leishmania - an aneuploid organism, can be performed as segregation tests that explore allele distribution, thus representing a good tool for population genetics, even for loci with undetermined ploidy. The bias of underestimated diversity could be diminished through a proper representation of the circulating strains, such that the greatest number of different alleles would be detected. A deeper knowledge over the karyotypes profiles of the different strains is of major importance.

\section{Acknowledgments}

We thank the Plataforma de Sequenciamento do Instituto Oswaldo Cruz (PDTIS) for performing all the sequencing; Instituto Oswaldo Cruz - FIOCRUZ, CNPq/Fiocruz (PIBIC) and Faperj for financial support. We also would like to thanks Global Science Editing, UK, Mariel Marlow and Hugo Caire Castro de Faria Neto for English revision.

\section{References}

Akopyants, N.S., Kimblin, N., Secundino, N., Patrick, R., Peters, N., et al., 2009. Demonstration of genetic exchange during cyclical development of Leishmania in the sand fly vector. Science 324, 265-268.

Alam, M.Z., Kuhls, K., Schweynoch, C., Sundar, S., Rijal, S., et al., 2009. Multilocus microsatellite typing (MLMT) reveals genetic homogeneity of Leishmania donovani strains in the Indian subcontinent. InfectGenetEvol 9, 24-31.

Belli, A.A., Miles, M.A., Kelly, J.M., 1994. A putative Leishmania panamensis/ Leishmania braziliensis hybrid is a causative agent of human cutaneous leishmaniasis in Nicaragua. Parasitology 109 (Pt. 4), 435-442.

Benatti, T.R., Valicente, F.H., Aggarwal, R., Zhao, C., Walling, J.G., et al., 2010. A neosex chromosome that drives postzygotic sex determination in the hessian fly (Mayetiola destructor). Genetics 184, 769-777.

Boité, M.C., Mauricio, I.L., Miles, M.A., Cupolillo, E., 2012. New insights on taxonomy, phylogeny and population genetics of Leishmania (Viannia) parasites based on multilocus sequence analysis. PLoS Negl. Trop. Dis. 6, e1888.

Boothroyd, C.E., Dreesen, O., Leonova, T., Ly, K.I., Figueiredo, L.M., et al., 2009. A yeast-endonuclease-generated DNA break induces antigenic switching in Trypanosoma brucei. Nature 459, 278-281.

Brito, M.E., Andrade, M.S., Mendonca, M.G., Silva, C.J., Almeida, E.L., et al., 2009. Species diversity of Leishmania (Viannia) parasites circulating in an endemic area for cutaneous leishmaniasis located in the Atlantic rainforest region of northeastern Brazil. Trop. Med. Int. Health 14, 1278-1286.

Camara, A.C., Varela-Freire, A.A., Valadares, H.M., Macedo, A.M., D’Avila, D.A., et al., 2010. Genetic analyses of Trypanosoma cruzi isolates from naturally infected triatomines and humans in northeastern Brazil. Acta Trop. 115, 205-211.

Coelho, A.C., Leprohon, P., Ouellette, M., 2012. Generation of Leishmania hybrids by whole genomic DNA transformation. PLoS Negl. Trop. Dis. 6, e1817.

Cupolillo, E., Grimaldi, G., Momen, H., 1994. A general classification of new-world Leishmania using numerical zymotaxonomy. Am. J. Trop. Med. Hyg. 50, 296311.

Dujardin, J.C., De Doncker, S., Jacquet, D., Banuls, A.L., Balavoine, M., et al., 2007. Clonal propagation and the fast generation of karyotype diversity: an in vitro Leishmania model. Parasitology 134, 33-39.

El Baidouri, F., Diancourt, L., Berry, V., Chevenet, F., Pratlong, F., et al., 2013. Genetic structure and evolution of the Leishmania genus in Africa and Eurasia: what does MLSA tell us. PLoS Negl. Trop. Dis. 7, e2255.

Ferreira, G.E., dos Santos, B.N., Dorval, M.E., Ramos, T.P., Porrozzi, R., et al., 2012. The genetic structure of Leishmania infantum populations in Brazil and its possible association with the transmission cycle of visceral leishmaniasis. PLoS One 7, e36242.

Garin, Y.J., Sulahian, A., Pratlong, F., Meneceur, P., Gangneux, J.P., et al., 2001. Virulence of Leishmania infantum is expressed as a clonal and dominant phenotype in experimental infections. Infect. Immun. 69, 7365-7373.

Gonzalez-Gonzalez, A., Sanchez-Reyes, L.L., Delgado Sapien, G., Eguiarte, L.E., Souza, V., 2013. Hierarchical clustering of genetic diversity associated to different levels of mutation and recombination in Escherichia coli: a study based on Mexican isolates. Infect. Genet. Evol. 13, 187-197.

Jinkerson, R.E., Radakovits, R., Posewitz, M.C., 2013. Genomic insights from the oleaginous model alga Nannochloropsis gaditana. Bioengineered 4, 37-43.

Martin, D.P., Lemey, P., Lott, M., Moulton, V., Posada, D., et al., 2010. RDP3: a flexible and fast computer program for analyzing recombination. Bioinformatics 26 , $2462-2463$.

Oddone, R., Schweynoch, C., Schonian, G., de Sousa, C.S., Cupolillo, E., et al., 2009. Development of a multilocus microsatellite typing approach for discriminating strains of Leishmania (Viannia) species. JClinMicrobiol 47, 2818-2825.

Otto, T.D., Vasconcellos, E.A., Gomes, L.H., Moreira, A.S., Degrave, W.M., et al., 2008 ChromaPipe: a pipeline for analysis, quality control and management for a DNA sequencing facility. Genet. Mol. Res. 7, 861-871. 
Paul, S., Linardopoulou, E.V., Billig, M., Tchesnokova, V., Price, L.B., et al., 2013. Role of homologous recombination in adaptive diversification of extraintestinal Escherichia coli. J. Bacteriol. 195, 231-242.

Prugnolle, F., De Meeus, T., 2010. Apparent high recombination rates in clonal parasitic organisms due to inappropriate sampling design. Heredity (Edinb.) 104, 135-140.

Rickert, A.M., Premstaller, A., Gebhardt, C., Oefner, P.J., 2002. Genotyping of Snps in a polyploid genome by pyrosequencing. Biotechniques 32 (592-593), 596-598, 600 passim.

Rogers, M.B., Hilley, J.D., Dickens, N.J., Wilkes, J., Bates, P.A., et al., 2011. Chromosome and gene copy number variation allow major structural change between species and strains of Leishmania. Genome Res. 21, 21292142.

Rougeron, V., De Meeus, T., Hide, M., Waleckx, E., Dereure, J., et al., 2010. A battery of 12 microsatellite markers for genetic analysis of the Leishmania (Viannia) guyanensis complex. Parasitology 137, 1879-1884.

Sterkers, Y., Lachaud, L., Crobu, L., Bastien, P., Pages, M., 2011. FISH analysis reveals aneuploidy and continual generation of chromosomal mosaicism in Leishmania major. Cell Microbiol. 13, 274-283.

Sterkers, Y., Lachaud, L., Bourgeois, N., Crobu, L., Bastien, P., et al., 2012. Novel insights into genome plasticity in Eukaryotes: mosaic aneuploidy in Leishmania. Mol. Microbiol. 86, 15-23.

Tamura, K, Peterson, D, Peterson, N., Stecher, G, Nei, M., et al, 2011. MEGA5: molecular evolutionary genetics analysis using maximum likelihood, evolutionary distance, and maximum parsimony methods. Mol. Biol. Evol. 28, 2731-2739.

Tibayrenc, M., Ayala, F.J., 2012. Reproductive clonality of pathogens: a perspective on pathogenic viruses, bacteria, fungi, and parasitic protozoa. Proc. Natl. Acad. Sci. U.S.A. 109, E3305-E3313.

Tibayrenc, M., Ayala, F.J., 2013. How clonal are Trypanosoma and Leishmania? Trends Parasitol. 29, 264-269.

Tibayrenc, M., Kjellberg, F., Ayala, F.J., 1990. A clonal theory of parasitic protozoa: the population structures of Entamoeba, Giardia, Leishmania, Naegleria, Plasmodium, Trichomonas, and Trypanosoma and their medical and taxonomical consequences. ProcNatlAcadSciUSA 87, 2414-2418.

Ubeda, J.M., Legare, D., Raymond, F., Ouameur, A.A., Boisvert, S., et al, 2008. Modulation of gene expression in drug resistant Leishmania is associated with gene amplification, gene deletion and chromosome aneuploidy. Genome Biol. 9 R115.

van Belkum, A., Struelens, M., de Visser, A., Verbrugh, H., Tibayrenc, M., 2001. Role of genomic typing in taxonomy, evolutionary genetics, and microbial epidemiology. Clin. Microbiol. Rev. 14, 547-560.

Vink, C., Rudenko, G., Seifert, H.S., 2011. Microbial antigenic variation mediated by homologous DNA recombination. FEMS Microbiol. Rev..

Zemanova, E., Jirku, M., Mauricio, I.L., Horak, A., Miles, M.A., et al., 2007. The Leishmania donovani complex: genotypes of five metabolic enzymes (ICD, ME, MPI, G6PDH, and FH), new targets for multilocus sequence typing. Int. J. Parasitol. 37, 149-160. 